\title{
Screw theory-based stiffness analysis for a fluidic-driven soft robotic manipulator
}

\author{
Jialei Shi ${ }^{1}$, Julio C. Frantz ${ }^{2}$, Azadeh Shariati ${ }^{1}$, Ali Shiva ${ }^{3}$ \\ Jian S Dai ${ }^{3}$, Daniel Martins ${ }^{2}$ and Helge A. Wurdemann ${ }^{1}$
}

\begin{abstract}
Soft robotic manipulators have been created and investigated for a number of applications due to their advantages over rigid robots. In minimally invasive surgery, for instance, soft robots have successfully demonstrated a number of benefits due to the compliant and flexible nature of the material they are made of. However, these type of robots struggle with performing tasks that require on-demand stiffness i.e. exerting higher forces to the surrounding environment. A number of semi-active and active mechanisms have been investigated to change and control the stiffness of soft robotic manipulators. Embedding these mechanisms in soft manipulators for spacerestricted applications can be challenging though.

To better understand the inherent passive stiffness properties of soft manipulators, we propose a screw theory-based stiffness analysis for fluidic-driven continuum soft robotic manipulators. First, we derive the forward kinematics based on a parameterbased piece-wise constant curvature model. It is worth noting, our stiffness analysis can be conducted based on any freespace forward kinematic model. Then our stiffness analysis and mapping methodology is conducted based on screw theory. Initial results of our approach demonstrate the feasibility comparing computational and experimental data.
\end{abstract}

\section{INTRODUCTION}

Soft robotics has established itself as an prevalent research topic for its versatility and intrinsic adaptability to unstructured environments [1]-[3]. Soft robotic structures are inherently flexible and compliant [4], [5] able to perform morphological transitions and anthropomorphic manipulation [6]. Actuation systems for soft robots can be classified into three categories: tendon-, fluidic- and electro-active polymerdriven actuation [7]. In particular, fluidic-driven robots are rather cost-efficient and have been extensively explored for applications in the industrial sector where robots work closely together with humans [8], [9], in minimally invasive interventions [10]-[13], and rehabilitation [14]. However, as a result of their inherently soft property, the ability to exert forces on the environment, when required, and effectively change the stiffness on demand is challenging and yet to be further explored [15]-[17].

A number of mechanisms have been investigated able to vary the stiffness of soft robotic manipulators. The main

This work is supported by the Springboard Award of the Academy of Medical Sciences (grant number: SBF003-1109), the UCL Dean's Prize, UCL Mechanical Engineering, and the China Scholarship Council (CSC).

${ }^{1}$ J. Shi, A. Shariati and H.A. Wurdemann are with the Department of Mechanical Engineering, University College London, UK. h. wurdemann@ucl.ac.uk

${ }^{2}$ J.C. Frantz and D. Martins are with the Department of Mechanical Engineering, Federal University of Santa Catarina, Brazil.

${ }^{3}$ A. Shiva and J.S. Dai are with the Department of Informatics, King's College London, UK. jian.dai@kcl.ac.uk

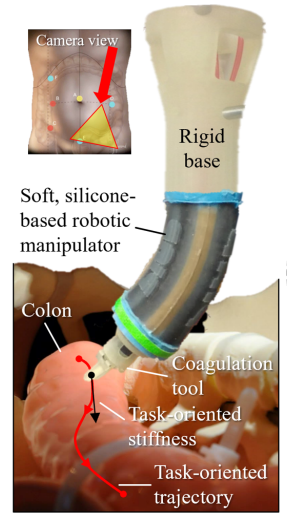

(a)

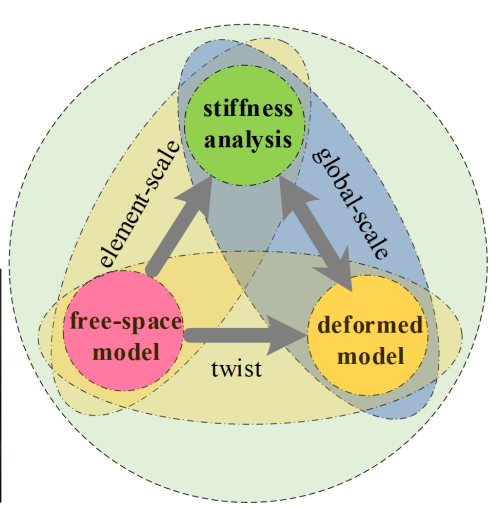

(b)
Fig. 1. Soft robotic manipulator and applied methodology: (a) This soft robotic manipulator has three embedded fluidic actuation chambers. We analysed the configuration of our soft robotic manipulator to understand stiffness in a task-specific direction. (b) Screw theory based modelling methodology schematics.

stiffening technologies can be classified as semi-active and active: On the one hand, materials can be embedded into soft robotic structure allowing to intrinsically tune the robot's stiffness [18], [19]. Active stiffening can be achieved by applying antagonistic actuation [20], [21]. In [22], stiffness mechanisms have been thoroughly reviewed.

However, embedding the aforementioned stiffness mechanisms in soft manipulators for space-restricted applications, for instance, minimally invasive surgery (MIS) can be challenging [19]. Here, a configuration-based stiffness analysis of the soft robot might be advantageous. In [23] for instance, a quasi-static model-based position and force estimation method of fluidic-driven robotic with variable stiffness under external forces is proposed. In [24], [25], a piece-wise constant strain model is presented based on the discrete Cosserat beam theory in which the manipulator state is described by a finite set of constant strains. Furthermore, further studies published in [26], [27] are based on traditional EulerBernoulli beam or 3D Timoshenko models which include stiffness parameters of the soft silicone material. Overall, the authors focussed on kinematic modelling using stiffness properties of the material of the manipulators including external forces rather than a detailed stiffness analysis. The application of screw theory can provide a unique insight to understand the stiffness of continuum-type robots. In [28], [29] for instance, the compliance characteristics of manipulator made of planar springs has been explored. 
A screw theory-based stiffness analysis is proposed in this paper with the focus on understanding configuration-based stiffness of fluidically actuated soft robotic manipulators for task-specific applications. Our model is applied to the STIFFFLOP manipulator made of a cylindrical-shaped, silicone body with three pairs of actuation chambers (Fig. 1). The main contribution of this paper lies in our stiffness-oriented modelling methodology for soft robots utilising screw theory. Our models is then implemented and verified based on a parameter-based piece-wise constant curvature (PCC) model. Besides, our stiffness analysis method can be conducted based on any free-space forward kinematic models of different manipulators.

In Section II, a detailed description of the continuum robot is given as well as the derivation of the proposed methodology. In Section III, simulation of the forward kinematics and stiffness analysis are conducted and compared to experimental data validating the effectiveness of the proposed method. Section IV summarises the conclusions and identifies the direction of future work.

\section{SCREW THEORY-BASED SOFT ROBOTIC KINEMATICS}

The soft robotic manipulator used in this paper is a modified version of STIFF-FLOP consisting of one cylindrical segment made of silicone material [Ecoflex 00-50 Supersoft, SmoothOn] with an overall length of $90 \mathrm{~mm}$, outer diameter of $30 \mathrm{~mm}$ [12]. The segment has 6 fibre-reinforced inflatable pneumatic chambers, each chamber has a diameter of $4.5 \mathrm{~mm}$ and evenly located in the periphery of the silicone cylinder as shown in Fig. 2. The adjacent two-chamber pairs are pneumatically actuated simultaneously via a $2 \mathrm{~mm}$ outer diameter inlet air pipe. Pressurisation of one or two chamber pairs results in bending while actuating all three chamber pairs at the same time will elongate the manipulator. Fig. 1(b) presents the overall methodology which is described in detail the following subsections.

\section{A. Screw theory notation}

The kinematics of the aforementioned soft manipulator is described using screw theory, an algebra method that can include exerted forces and motions [30]. There are two fundamental screws comprising infinitesimal displacement and motion called twist and wrench, respectively:

1) Twist: Twist $\$_{M}$ written in Plücker axis coordinates is defined by a straight line with an associated pitch $h$ as in (1).

$$
\$_{M}=\left[\begin{array}{c}
\Delta \delta \\
-- \\
\Delta \theta
\end{array}\right]=\left[\begin{array}{c}
-\vec{\omega} \times \vec{S}_{0}+h \vec{\omega} \\
----- \\
\vec{\omega}
\end{array}\right]=\left[\begin{array}{c}
\vec{v} \\
-- \\
\vec{\omega}
\end{array}\right] \in \mathbb{R}^{6}
$$

where $\Delta \delta=\left[\Delta \delta_{x}, \Delta \delta_{y}, \Delta \delta_{z}\right]^{T}$ is a $3 \times 1$ translational displacement vector, $\Delta \theta=\left[\Delta \theta_{x}, \Delta \theta_{y}, \Delta \theta_{z}\right]^{T}$ is a $3 \times 1$ rotational displacement vector. $\vec{\omega}$ denotes direction ratios pointing along the screw axis, $\vec{S}_{0}$ is the position vector of any point on the screw axis with respect to the reference system. The twist vector could also be written in the form of an isomorphism matrix in Lie algebra $\mathfrak{s e}(3)$ as in (2).

$$
\hat{\$}_{M}=\left[\begin{array}{cc}
\hat{\vec{\omega}} & \vec{v} \\
0_{[1 \times 3]} & 0
\end{array}\right] \in \mathfrak{s e}(3), \hat{\vec{\omega}}=\left[\begin{array}{ccc}
0 & -\omega_{3} & \omega_{2} \\
\omega_{3} & 0 & -\omega_{1} \\
-\omega_{2} & \omega_{1} & 0
\end{array}\right]
$$

The transformation matrix $T$ can be obtained by taking the exponential of $\hat{\$} \theta$ as in (3).

$$
T=e^{\hat{\$} \theta}=\left[\begin{array}{cc}
R_{[3 \times 3]} & p_{[3 \times 1]} \\
0_{[1 \times 3]} & 1
\end{array}\right] \in \mathrm{SE}(3), \mathrm{R} \in \mathrm{SO}(3), \mathrm{p} \in \mathbb{R}^{3}
$$

Here, $R$ is a rotation matrix, $p$ is a translation vector and $\theta$ a rotation angle. In this way, the derived configuration space $g(t)=T g_{t}(0), g_{t}(0)$ result in the initial position matrix.

2) Wrench: Wrench $\$_{W}$ written in Plücker ray coordinates is defined in a similar way shown in (4).

$$
\$_{W}=\left[\begin{array}{c}
\vec{F} \\
\vec{S}_{0} \times \vec{F}+h \vec{F}
\end{array}\right]=\left[\begin{array}{c}
\mathbf{F} \\
\mathbf{M}
\end{array}\right]
$$

Here, $\vec{S}_{0}$ is the orientation vector of the wrench, $\mathbf{F}=$ $\left[f_{x}, f_{y}, f_{z}\right]$ is the 3 applied force and $\mathbf{M}=\left[M_{x}, M_{y}, M_{z}\right]$ is the $3 \times 1$ applied torque.

3) Screw coordinate transformation: Considering two screws $\$_{a}, \$_{b}$, both are in the form of Plücker ray coordinate. $\$_{a}$ and $\$_{b}$ are written in Cartesian frames $a$ and $b$, respectively. In such circumstances, $6 \times 6$ adjoint transformation screws $\operatorname{Ad}_{g}$ can be defined between those two frames. The adjoint matrix $\operatorname{Ad}_{a b}$ transforms screw $\$_{a}$ to $\$_{b}$

$$
\operatorname{Ad}_{a b}=\left[\begin{array}{cc}
R_{a b} & 0_{[3 \times 3]} \\
\hat{p}_{a b} R_{a b} & R_{a b}
\end{array}\right]
$$

where $p_{a b}$ is the translational vector and $R_{a b}$ is rotational vector, $\hat{p}_{a b}$ is written in the form of (2).

\section{B. Free-space kinematics}

1) Kinematics of each discrete element: Without any external forces applied to the soft manipulator, the PCC method is feasible for quasi-static modelling of our continumm robot [31]. Hence, a parameterised PCC model is derived within the context of screw theory framework as shown in Fig. 2. One discrete arc can be described using five equivalent joints which are depicted by five twists whose parameters are summarised in Table I.

The discretised element $t^{t h}$ spatial curve is described using the three parameters $(\kappa, s, \phi)$, where $\kappa$ is the curve ratio, $s$ is the curve length and $\phi$ the rotation about the $z$-axis. Equation (6) yields from Fig. 2.

$$
\theta_{1}^{i}=-\theta_{5}^{i}=\phi^{i}, \theta_{2}^{i}=\theta_{4}^{i}=\frac{\kappa^{i} s^{i}}{2}, d_{3}^{i}=\frac{2 \sin \left(\kappa^{i} s^{i} / 2\right)}{\kappa^{i}}
$$

\begin{tabular}{|c|c|c|c|c|c|c|c|}
\hline Link & \multicolumn{3}{|c|}{$v$} & \multicolumn{3}{|c|}{$\omega$} & Amplitude \\
\hline 1 & 0 & 0 & 0 & 0 & 0 & 1 & $\theta_{1}^{i}=\phi^{i}$ \\
\hline 2 & 0 & 0 & 0 & 0 & 1 & 0 & $\theta_{2}^{i}=\kappa^{i} s^{i} / 2$ \\
\hline 3 & 0 & 0 & 1 & 0 & 0 & 0 & $d_{3}^{i}=\left(2 / k^{i}\right) \sin \kappa^{i} s^{i} / 2$ \\
\hline 4 & 0 & 0 & 0 & 0 & 1 & 0 & $\theta_{4}^{i}=\kappa^{i} s^{i} / 2$ \\
\hline 5 & 0 & 0 & 0 & 0 & 0 & 1 & $\theta_{5}^{i}=-\phi^{i}$ \\
\hline
\end{tabular}

TABLE I

SCREW PARAMETERS FOR THE SOFT ROBOTIC MANIPULATOR BASED ON THE CHAIN SHOWN IN FIGURE 2. 
Compared to the Denavit-Hartenberg conversion used in [31], a more elaborate transformation frame analysis is replaced by a product of exponentials eliminating the need of link frames here. Then the transformation matrix yields in (7) in line with [31].

$$
\begin{aligned}
& \bar{T}_{(i-1) i}=e^{\hat{\xi_{1}} \theta_{1}} e^{\hat{\xi_{2}} \theta_{2}} e^{\hat{\xi}_{3} d_{3}} e^{\hat{\xi_{4}} \theta_{4}} e^{\xi_{5} \hat{\theta}_{5}} \\
& =\left[\begin{array}{r}
-s_{1} s_{5}+c_{1} c_{2} c_{4} c_{5}-c_{1} s_{2} s_{4} c_{5} \\
c_{1} s_{5}+s_{1} c_{2} c_{4} c_{5}-s_{1} s_{2} s_{4} c_{5} \\
-s_{24} c_{5} \\
0
\end{array}\right. \\
& \left.\begin{array}{rrr}
-s_{1} c_{5}+c_{1} s_{2} s_{4} s_{5}-c_{1} c_{2} c_{4} s_{5} & s_{24} c_{1} & c_{1} s_{2} d_{3} \\
c_{1} c_{5}+s_{1} s_{2} s_{4} s_{5}-s_{1} c_{2} c_{4} s_{5} & s_{24} s_{1} & s_{1} s_{2} d_{3} \\
s_{24} s_{5} & c_{24} & c_{2} d_{3} \\
0 & 0 & 1
\end{array}\right] \\
& =\left[\begin{array}{cccc}
c_{\phi}^{2}\left(c_{(\kappa s)}-1\right)+1 & s_{\phi} c_{\phi}\left(c_{(\kappa s)}-1\right) & c_{\phi} s_{(\kappa s)} & \frac{c_{\phi}\left(1-c_{(\kappa s)}\right)}{\kappa} \\
s_{\phi} c_{\phi}\left(c_{(\kappa s)}-1\right) & c_{\phi}^{2}\left(1-c_{(\kappa s)}\right)+c_{(\kappa s)} & s_{\phi} s_{(\kappa s)} & \frac{s_{\phi}\left(1-c_{(\kappa s)}\right)}{\kappa} \\
-c_{\phi} s_{(\kappa s)} & -s_{\phi} s_{(\kappa s)} & c_{(\kappa s)} & \frac{s_{(\kappa s)}}{\kappa} \\
0 & 0 & 0 & 1
\end{array}\right]
\end{aligned}
$$

where $c_{i}=\cos i, s_{i}=\sin i$ and $c_{i j}=\cos (i+j), s_{i j}=\sin (i+j)$ with numerical subscripts. Finally, the transformation matrix $\bar{T}_{(i-1) i}$ of a spatial arc is presented by $\left(\kappa^{i}, s^{i}, \phi^{i}\right)$.

2) Internal wrench: The internal wrench is generated by pressurized chambers. Moments about the $x$ - and $y$-axis resulting in bending motions and the $z$-axis force resulting in elongation can be represented as a wrench in screw form, which can be written as $\$_{W_{i}}=\left[f_{x i}, f_{y i}, f_{z i}, m_{x i}, m_{y i}, m_{z i}\right]^{T}$ where $i=1, \ldots, 6$ presents the number of chambers. Defining the distance between the chamber centre to the origin as $r_{c_{i}}$ and the angle $\alpha_{1}$ between two adjacent chambers, the wrench in chamber can be calculated as $i$ is $\$_{W_{p_{1}}}^{i}=\left[\begin{array}{ll}\mathbf{F} & \mathbf{M}\end{array}\right]^{T}=$ $\left[0,0, f_{z 1}, 0, r_{c_{i}} \times f_{z 1}, 0\right]^{T}$. As shown in Fig. 2, the $x$-axis is between the first two chamber pairs. Then, the position vectors of the result of chambers can be obtained by rotation

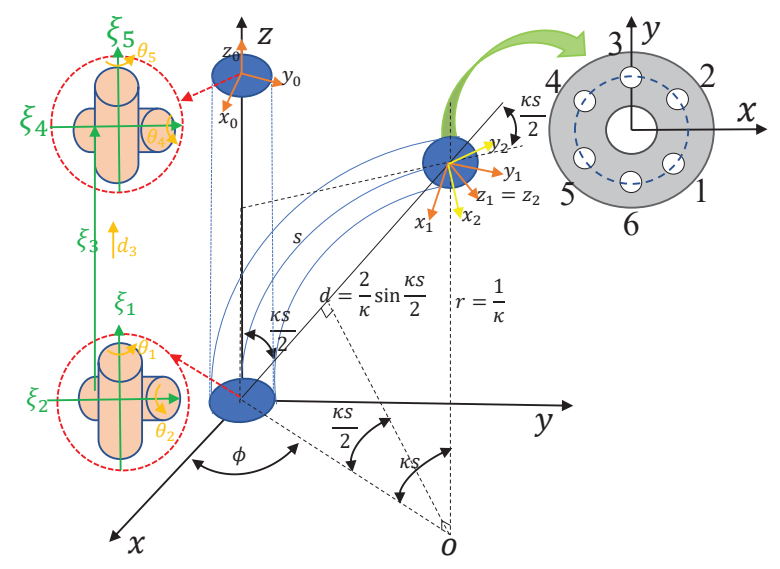

Fig. 2. Geometrical relationship and kinematic chain description: One arc section is described using 5 twists $\xi_{i}(i=1,2,3,4,5)$ with screw axes $(z, y, z, y, z)$ and amplitudes $\left(\theta_{1}, \theta_{2}, d_{3}, \theta_{4}, \theta_{5}\right)$. Note that, for simplification, segment index $i$ is neglected here. The initial frame is $\left(x_{0}, y_{0}, z_{0}\right)$, with the tip frame being $\left(x_{1}, y_{1}, z_{1}\right)$. $\left[r_{c_{1}}, 0,0\right]^{T}$ about the $z$-axis using the well-known rotation matrix $R_{z}\left(\phi_{i}\right)$ in (8).

$$
R_{z}\left(\phi_{i}\right)=\left[\begin{array}{ccc}
\cos \left(\phi_{i}\right) & -\sin \left(\phi_{i}\right) & 0 \\
\sin \left(\phi_{i}\right) & \cos \left(\phi_{i}\right) & 0 \\
0 & 0 & 1
\end{array}\right]
$$

where $\phi_{i}$ equals to

$$
\phi_{i}=-\frac{\alpha_{1}}{2}+\frac{i-i \% 2}{2} \alpha_{1}+\frac{(i-1)-(i-1) \% 2}{2}\left(120^{\circ}-\alpha_{1}\right)
$$

where $\%$ is the modulus operator, $i=1 \ldots 6, \alpha_{1}=60^{\circ}$. This yields chamber vector $S_{0_{i}}=\left[x_{i}, y_{i}, 0\right]^{T}=R_{z}\left(\phi_{i}\right) S_{0_{1}}$.

To compute the forces $f_{z i}$ in each chamber pair, it is necessary to apply $f_{z i}=P_{i} A_{c}=P_{i} \pi R_{p}^{2} . P_{i}$ represents the pressure in each chamber, $R_{p}$ is the radius of chamber and $A_{c}$ is the cross-sectional area of each pressurised chamber. In line with the above procedure, all wrenches of all individual chambers can be calculated. The equivalent wrench $\$_{W_{p}}^{i}$ written in body frame of the $i^{\text {th }}$ element is obtained by summing them as in $\$_{W_{p}}^{i}=\sum_{j=1}^{6} \$_{W_{p_{j}}}^{i}=\left[F_{x_{p}}^{i}, F_{y_{p}}^{i}, F_{z_{p}}^{i}, M_{x_{p}}^{i}, M_{y_{p}}^{i}, M_{z_{p}}^{i}\right]^{T}$.

Now, it is possible to substitute the wrenches for the arc parameters. Firstly, the curvatures in the $x-z$ and $y-z$ plane can be calculated as $\kappa_{x}^{i}=M_{x_{p}} /\left(E I_{x}\right), \kappa_{y}^{i}=M_{y_{p}} /\left(E I_{y}\right)$. The second moments of the area $I_{x}$ yields in

$$
I_{x}=\frac{\pi R_{o}^{4}}{4}-\frac{\pi R_{i}^{4}}{4}-\sum_{i=1}^{6}\left(\frac{\pi R_{p}^{4}}{4}+y_{i}^{2} \pi R_{p}^{2}\right)
$$

where $R_{o}$ is the outer radius, $R_{i}$ is the inner radius, and $y_{i}$ the distance related to $x$ - axis of each chamber. $I_{y}$ can be calculated in the same way. It is evident that $I_{x}$ and $I_{y}$ are equal to $I$ written in the frame shown in Fig. 2. The curvature of the soft robot is now calculated as $\kappa_{p}^{i}=\sqrt{\left(\kappa_{x}^{i}\right)^{2}+\left(\kappa_{y}^{i}\right)^{2}}$. So, the rotational angle $\phi^{i}$ around the $z$-axis is calculated by $\phi_{p}^{i}=-\operatorname{atan} 2\left(\kappa_{x}^{i}, \kappa_{y}^{i}\right)$.

Considering a total elongation $\delta_{z}=\left(f_{z} l\right) /(E A)$ and $A=$ $\pi\left(R_{o}^{2}-R_{i}^{2}-6 \pi R_{p}^{2}\right)$ being the cross-sectional area, the length of each discrete segment can be calculated as $s_{p}^{i}=\left(\delta_{z}^{i}+l^{i}\right) / n$. It is worth noting that the the cross-sectional area will decrease due to elongation influencing the values of $A, I$. In addition, a larger deformation of the hyper-elastic material will result in non-linear strain-stress ranges of the material which means that $E$ will change. In fact, $A, I$ will not only change, but also the production of $E I$ which again influences $E A$ and the modelling accuracy. In order to compensate such non-linearity, three pressure-dependent compensation parameters $\lambda_{E}\left(P_{i}\right), \lambda_{A}\left(P_{i}\right), \lambda_{I}\left(P_{i}\right)$ are introduced to improve the accuracy of traditional PCC method. Those values are adjustable coefficients of the nominal system parameters. Finally, the continuum robot is modelled using $i$ discrete elements as shown in Fig. 2. In this way, the arc parameters $\left(\kappa^{i}, \phi^{i}, s^{i}\right)^{T}$ can be obtained and substituted into (7). The complete free-space forward kinematics can now be derived by exponential integration along the soft manipulator.

\section{Spatial stiffness modelling and analysis}

The stiffness properties of the manipulator will change depending on different configurations resulting from various 
chamber pressurisation. Combined with the established kinematics model, the manipulator's stiffness can be calculated iteratively. Here, we demonstrate how to derive spatial stiffness using screw theory illustrated in Fig. 3.

Firstly, the external wrench $\$_{W_{e}}^{t}=\left[F_{x}^{i}, F_{y}^{i}, F_{z}^{i}, M_{x}^{i}, M_{y}^{i}, M_{z}^{i}\right]$ written in the tip frame is applied to the tip. It is convenient to conduct an analysis in backward direction from $s=l \rightarrow 0$. Considering the screw coordinate transformation in (5), the transmitted body wrench at any $i^{\text {th }}$ position written in the tip frame $\$_{W_{e}}^{i}$ can be obtained by iterative numerical calculation from the tip to the current element.

$$
\begin{aligned}
& \$_{W_{e}}^{i}=\operatorname{Ad}_{(i-1) i}^{-1} \$_{W_{e}}^{i-1}=\left[\begin{array}{cc}
R_{(i-1) i}^{T} & 0 \\
-R_{(i-1) i} \hat{p}_{(i-1) i} & R_{(i-1) i}^{T}
\end{array}\right] \$_{W_{e}}^{i-1} \\
& =\operatorname{Ad}_{(i-1) i}^{-1} \operatorname{Ad}_{(i-2)(i-1)}^{-1} \$_{W_{e}}^{i-2} \\
& =\left[\begin{array}{cc}
R_{(i-1) i}^{T} & 0 \\
-R_{(i-1) i} \hat{p}_{(i-1) i} & R_{(i-1) i}^{T}
\end{array}\right] * \\
& {\left[\begin{array}{cc}
R_{(i-2)(i-1)}^{T} & 0 \\
-R_{(i-2)(i-1)} \hat{p}_{(i-2)(i-1)} & R_{(i-2)(i-1)}^{T}
\end{array}\right] \$_{W_{e}}^{i-2}} \\
& =[\cdots][\cdots][\cdots] \$_{W_{e}}^{i-3}=\cdots \cdots \\
& =\left(\operatorname{Ad}_{t(t+1)} \operatorname{Ad}_{(t+1)(t+2)} \cdots \operatorname{Ad}_{(i-2)(i-1)} \operatorname{Ad}_{(i-1) i}\right)^{-1} \$_{W_{e}}^{t} \\
& =\mathrm{Ad}_{t i}^{-1} \$_{W_{e}}^{t}=\left[\begin{array}{cc}
R_{t i}^{T} & 0 \\
-R_{t i} \hat{p_{t i}} & R_{t i}^{T}
\end{array}\right] \$_{W_{e}}^{t}
\end{aligned}
$$

where $i j$ refers to the $3 \times 1$ translational vector $p_{i j}$, the $3 \times$ 3 rotatory matrix $R_{i j}$ or corresponding to the $6 \times 6$ adjoint matrix from the $i^{t h}$ to $j^{\text {th }}$ segment, and $\mathrm{Ad}^{-1}$ is the inverse adjoint matrix.

The twist $\$_{M_{e}}^{i}$ equals to infinitesimal deformation $\delta_{i}$ defined by (1) in each discretised element resulting from the applied wrench $\$_{W_{e}}^{i}$ by

$$
\begin{aligned}
& \$_{M_{e}}^{i}=\left[\begin{array}{llllll}
\Delta x^{i} & \Delta y^{i} & \Delta z^{i} & \Delta \theta_{x}^{i} & \Delta \theta_{y}^{i} & \Delta \theta_{z}^{i}
\end{array}\right]^{T} \\
& =\mathbf{K}^{i-1} \$_{W_{e}}^{i}=\mathbf{C}^{i} \$_{W_{e}}^{i}
\end{aligned}
$$

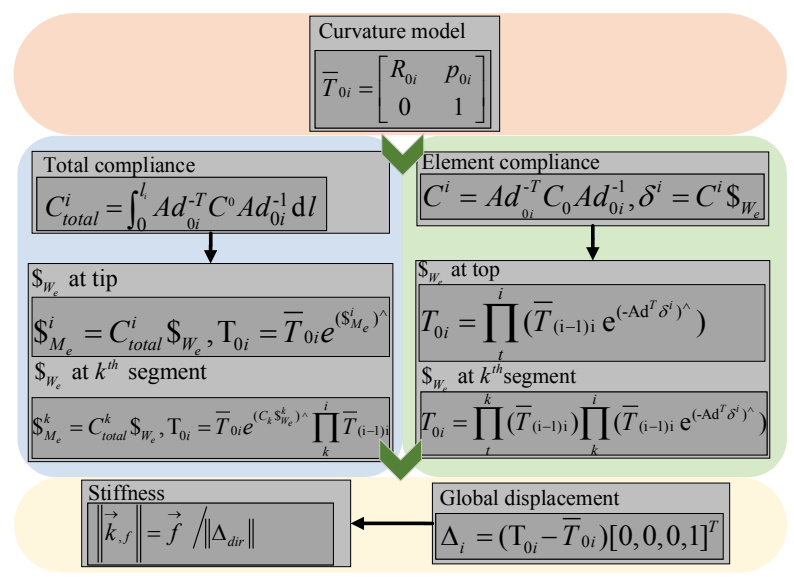

Fig. 3. Stiffness analysis scheme: Firstly, the compliance matrix (see (18)) is calculated (left). Then, the stiffness of each element is calculated based on (19) (right). Those two methods can be applied to free space forward kinematic models, e.g., the one presented in Section II. where $\mathbf{K}^{i} \in \mathbb{R}^{6 \times 6}$ is defined as our stiffness matrix. Furthermore, the inverse of matrix $\mathbf{K}^{i}$ is the compliance matrix $\mathbf{C}^{i}$.

Based on (5), the wrench transformation matrix from frame $a$ to frame $b$ can be written as $W_{a}=\operatorname{Ad}_{a b} W_{b}$. The corresponding deformation twist has the following relationship [32]:

$$
\mathscr{F} \$_{M_{a}}=\operatorname{Ad}_{a b}\left(\mathscr{F} \$_{M_{b}}\right)
$$

where $\mathscr{F}$ is elliptical polar operator which equals to

$$
\mathscr{F}=\left[\begin{array}{ll}
0_{[3 \times 3]} & \mathbf{I}_{[3 \times 3]} \\
\mathbf{I}_{[3 \times 3]} & 0_{[3 \times 3]}
\end{array}\right]
$$

Then, Equation (13) can be rewritten as

$\$_{M_{b}}=\left(\mathscr{F} \operatorname{Ad}_{a b} \mathscr{F}\right)^{-1} \$_{M_{a}}=\operatorname{Ad}_{a b}^{T} \$_{M_{a}}=C_{b} W_{b}=C_{b} \operatorname{Ad}_{a b}^{-1} W_{a}$

which can be simplified as $\$_{M_{a}}=\operatorname{Ad}_{a b}^{-T} C_{b} \operatorname{Ad}_{a b}^{-1} W_{a}$. All adjoint matrices are derived from pressurised kinematic model established in Section II. Then, the stiffness/compliance transformation relationship between two different frames can be summarised as

$$
\left\{\begin{array}{l}
C_{a}=\operatorname{Ad}_{a b}^{-T} C_{b} \operatorname{Ad}_{a b}^{-1} \\
K_{a}=C_{a}^{-1}=\operatorname{Ad}_{a b} K_{b} \operatorname{Ad}_{a b}^{T}
\end{array}\right.
$$

The compliance matrix of element $\mathbf{C}^{0}$ can be written as $\operatorname{diag}\left(G A, G A, E A, E I_{x}, E I_{y}, J I_{z}\right)^{-1}$. Based on (16), the spatial stiffness matrix of the $1^{\text {th }}$ element written in the tip frame is $\operatorname{Ad}_{t i}^{-T} \operatorname{diag}\left(G A, G A, E A, E I_{x}, E I_{y}, J I_{z}\right)^{-1} \mathrm{Ad}_{t i}^{-1}$.

Considering the discretised element with the arc length $s_{p}^{i}$, the corresponding element twist is calculated as $\delta_{i}=$ $\left(\mathrm{Ad}_{0 i}^{-T} C^{0} \mathrm{Ad}_{0 i}^{-1}\right) \$_{W_{e}}^{0} s_{p}^{i}$ and the body twist is $\operatorname{Ad}_{(i-1)(i)}^{T} \delta_{i}$. The continuum robot can be regarded as a serial mechanism i.e. the total deformation twist is the aggregation of each element [33]. The total deformation is derived as

$$
\Delta \$_{M_{e}}^{t}=\int_{0}^{l} \delta^{i} \mathrm{~d} s=\int_{0}^{l}\left(\operatorname{Ad}_{t i}^{-T} C^{0} \mathrm{Ad}_{t i}^{-1}\right) \$_{W_{e}} \mathrm{~d} s=C_{t} \$_{W_{e}}
$$

where $C_{t}$ is the $6 \times 6$ total stiffness matrix integrated from the tip to base. Equation (17) indicates that, when the wrench is an infinitesimal value, the deformed tip position can be calculated by $\bar{T}_{0 t} e^{\Delta \hat{\$}_{M_{e}}^{t}} . \bar{T}_{0 t}$ is the free-space transformation matrix. If a wrench force is exerted to the tip, (11) and (17) can be combined and the general deflection model for the $k^{\text {th }}$ segment can be written as

$$
\begin{aligned}
T_{0 k} & =\bar{T}_{0 k} e^{\Delta \hat{\$}_{M_{e}}^{k}} \\
& =\bar{T}_{0 k} e^{\left(\int_{0}^{l^{k}}\left(\operatorname{Ad}_{k i}^{-T} C^{0} \operatorname{Ad}_{k i}^{-1}\right) \operatorname{Ad}_{t k}^{-1} \$_{W e} \mathrm{~d} s\right)^{-}}=\bar{T}_{0 k} e^{\left(C_{k} \$_{W_{e}}^{k}\right)^{\curlywedge}}
\end{aligned}
$$

Furthermore, this model can also be interpreted by the discretised element from the tip to base. The updated stiffnessbased model can then be depicted as

$$
\begin{cases}T_{0 i}=\prod_{0}^{k}\left(\bar{T}_{(i-1) i}\right) \prod_{k}^{i}\left(\bar{T}_{(i-1) i} e^{\left(-\operatorname{Ad}_{t i}^{T} \delta^{i}\right)^{\curlywedge}}\right. & i>k, \$_{W_{e}} \text { at } k^{t h} \\ T_{0 i}=\prod_{0}^{i}\left(\bar{T}_{(i-1) i} e^{\left(-\mathrm{Ad}_{t i}^{T} \delta^{i}\right)^{\curlywedge}}\right. & \$_{W_{e}} \text { at tip }\end{cases}
$$


TABLE II

SYSTEM PARAMETERS FOR THE SOFT ROBOTIC MANIPULATOR

\begin{tabular}{cll|cll} 
symbol & unit & value & symbol & unit & value \\
\hline$r_{c}$ & $\mathrm{~mm}$ & 10.1 & $R_{p}$ & $\mathrm{~mm}$ & 15 \\
$R_{p}$ & $\mathrm{~mm}$ & 2.25 & $A_{c}$ & $\mathrm{~mm}^{2}$ & 31.81 \\
$E$ & $\mathrm{kPa}$ & 100 & $I_{x}$ & $\mathrm{~m}^{4}$ & $3.44 \times 10^{-8}$ \\
$I_{y}$ & $\mathrm{~m}^{4}$ & $3.44 \times 10^{-8}$ & $l$ & $\mathrm{~mm}$ & 90 \\
$R_{i}$ & $\mathrm{~mm}$ & 4.5 & $A$ & 547.8 & $\mathrm{~mm}^{2}$
\end{tabular}

where the inverse of (19) produced the same result as (18). Equation (19) generally is the stiffness-based modelling method. Without any pressurisation, (18) will yield in the results presented in [27].

\section{COMPUTATIONAL AND EXPERIMENTAL VALIDATION}

Numerical simulations and experiments will validate the feasibility of our methodology presented in Section II. The nominal system parameters of the soft robot are given in Table II. The compensation coefficients have been empirically chosen, so that the error of kinematics model is minimal, resulting in $\lambda_{E}=-0.2 \sqrt{p_{1}^{2}+p_{2}^{2}+p_{3}^{2}}+E, \lambda_{A}=1.2 A, \lambda_{I}=$ $1.05 I$ in this paper.

\section{A. Experimental protocol}

An NI-DAQ 6341 device is used to collect real-time pressure information and generate the PWM signals to control pressurised air supplied to different chambers by three individual pressure regulators (Camozzi K8P) supplied by a compressor (BAMBI MD Range Model 150/500). $x, y$ - and $z$-positions were recorded by an NDI Aurora electromagnetic trackers - a 6 DOF magnetic sensor was mounted at the tip of robot. An IIT-FT17 F/T (Force/Torque) sensor was attached to a motorised linear rail (Zaber X-LSM100A) to measure the stiffness $k$ in different direction and configuration using force $f$ and displacement data $\Delta x$ utilising $f=k \Delta x$. The setup shown in Fig. 4 is integrated into Matlab.

Two sets of experiments were carried out to validate the kinematics (Experiment 1) and stiffness validation and analysis (Experiment 2):

Experiment 1: To validate the kinematic model, $x, y$ and $z$-positions computed by our model are compared to experimental results when each chamber pair is linearly pressurised with $0.4,0.8$ and 1 .bar.

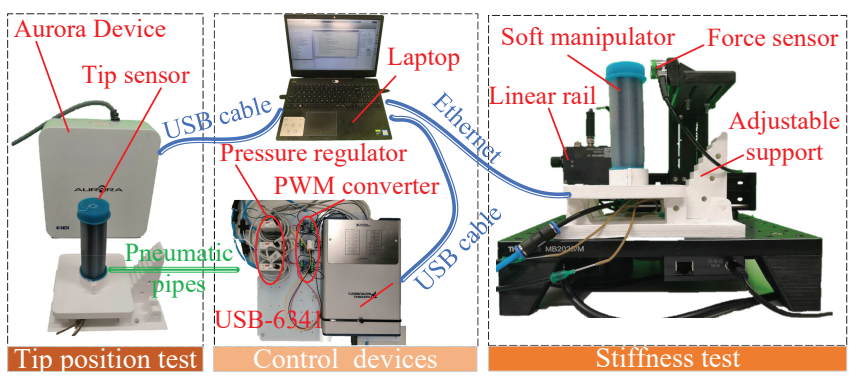

Fig. 4. The experimental setup includes control devices and the Aurora tracking system: When the chambers are pressurised, the tip position is recorded. Stiffness tests are conducted using a force sensor mounted on a motorised linear rail that displace the manipulator's tip.
Experiment 2: The stiffness of the manipulator was experimentally measured and validated with the numerical simulation based on the presented methodology with input pressures ranging from $0-1.5 \mathrm{bar}$. Then, the general stiffness mapping along the manipulator with different pressurisation in $x, y$ - and $z$-axis were analysed.

\section{B. Results and discussion}

A set of sawtooth pressure sequences is generated from $0-40 \mathrm{~s}$ as shown in Fig. 5(a). Fig. 5(b) shows the results of Experiment 1. It is worth noting that position data in all figures are tip positions in the base frame of the Aurora tracking system. All experiments are carried out five times. The top three graphs in Fig. 5(b) show the $x, y, z$ tip position of the experiments (in blue, green and yellow) and the simulation (in red). In the bottom graph of Fig. 5(b), the overall error is computed between the tip position in the experiments and simulation. The error region in three directions can be regarded within $5 \mathrm{~mm}$ which is around $5.5 \%$ of the entire length of the manipulator. It also can be observed that the maximum error is along $y$-axis at around $10 \mathrm{~mm}$, namely $10 \%$ (see $y$-position during $31-37 \mathrm{~s}$ ) which mainly results from the choices of simulation parameters. It is worth noting that the combination of compensation coefficients can be further optimised to mitigate the errors.

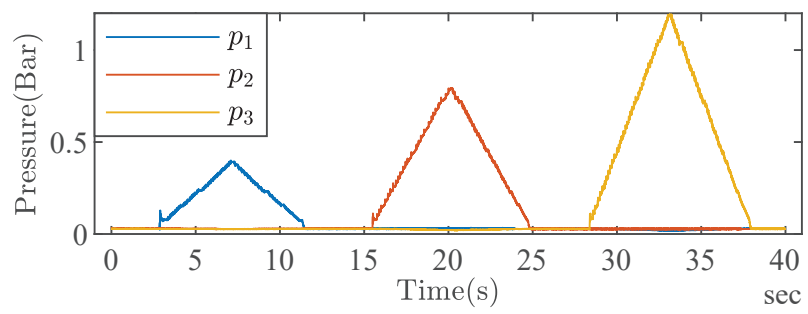

(a)
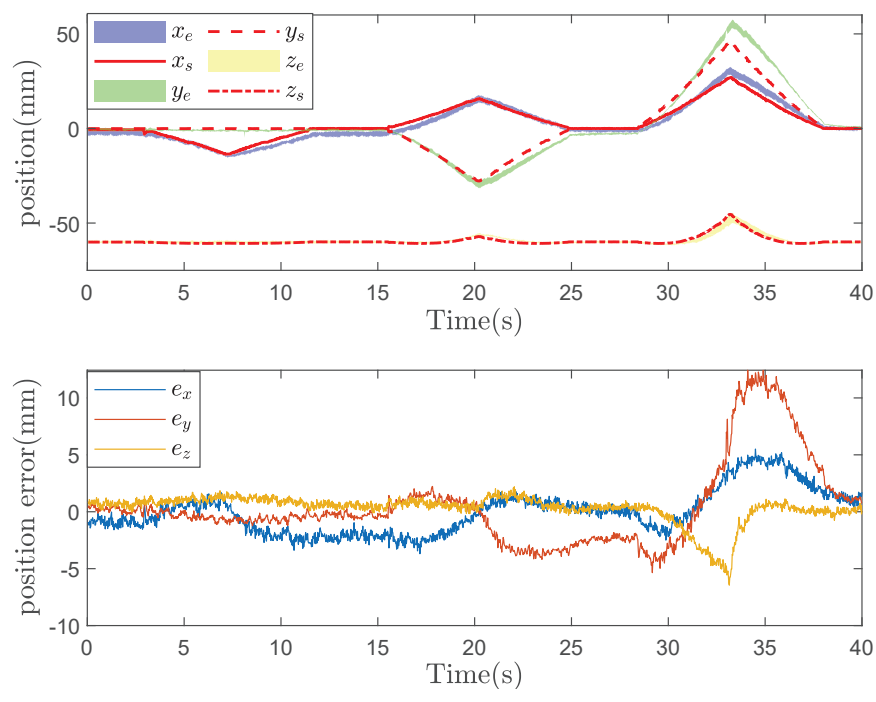

(b)

Fig. 5. Results of Experiment 1: (a) Input pressure values $p_{1}, p_{2}, p_{3}$ for each chamber pair. (b) Comparison between the simulation (line) and experimental (coloured shadow) data of the $x, y, z$ tip position. $x_{e}, y_{e}, z_{e}$ are experimental positions whereas $e_{x}, e_{y}, e_{z}$ are simulation outputs of the manipulator's tip and simulation errors in three directions. 

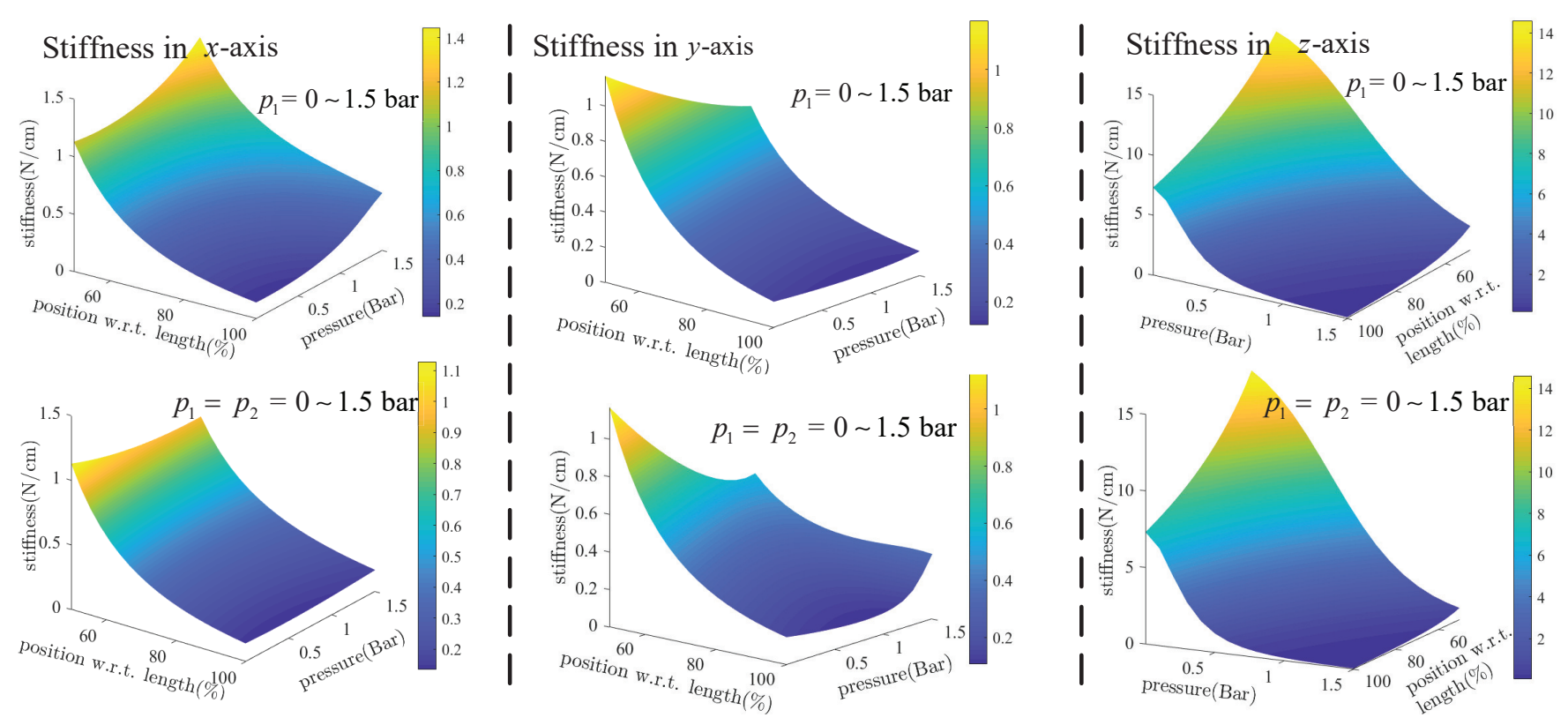

Fig. 6. Cartesian stiffness mapping prediction based on the protocol in Fig. 3: Planar stiffness along manipulator is plotted when one chamber pair and two chamber pairs are pressurised separately in $x, y$ and $z$-direction.

The experimental stiffness values (blue) of five repetitions and simulation (red) calculations for Experiment 2 are plotted in Fig. 7. One chamber pair is pressurised from $0-1.5$ bar and the global stiffness in $x$-,y- and $z$-direction is plotted. The experimental results show that the stiffness in $x$-direction increases from $0.19 \pm 0.03 \frac{\mathrm{N}}{\mathrm{cm}}$ to a maximum value of $0.59 \pm 0.045 \frac{\mathrm{N}}{\mathrm{cm}}$ with pressurisation from 0 bar to 1.5 bar. The stiffness in $y$-direction is almost not influenced by pressurisation and remains within $0.17 \pm 0.05 \frac{\mathrm{N}}{\mathrm{cm}}$. The stiffness in $z$-direction shows that values decrease from $11.1 \pm 0.04 \frac{\mathrm{N}}{\mathrm{cm}}$ to $0.18 \pm 0.03 \frac{\mathrm{N}}{\mathrm{cm}}$. It is worth noting the stiffness discrepancy between the simulation and experiments. For instance, the maximum stiffness error is about $1 \frac{\mathrm{N}}{\mathrm{cm}}$ in
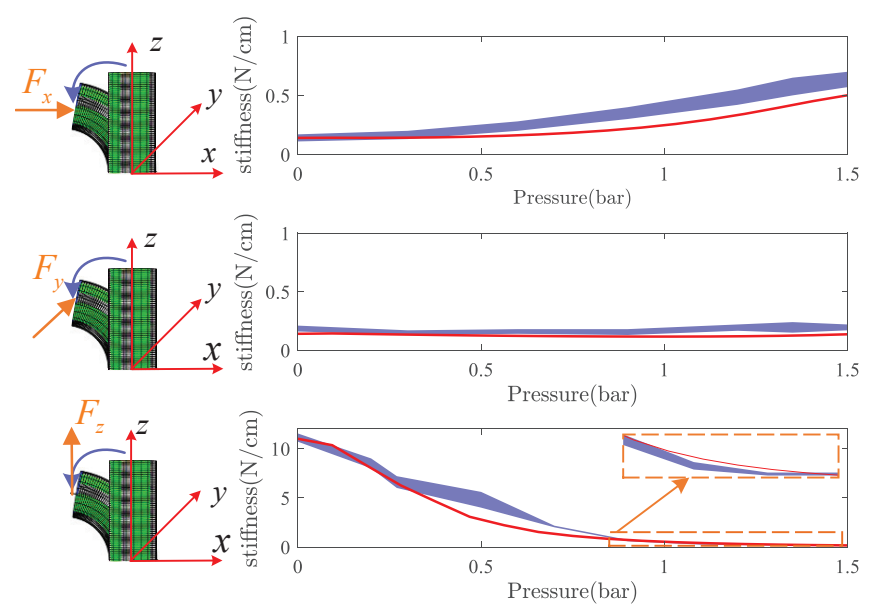

Fig. 7. Comparison of the experimental stiffness (blue) and simulation (red) results in 3 directions: One chamber pair is pressurised from 0-1.5 bar and the global stiffness in $x, y$ and $z$-direction is plotted. The blue arrow indicates the direction of deformation under the pressurisation. $z$-direction, which might result from the error propagation of the forward kinematics. The accuracy could be further improved by optimising the kinematics model.

Furthermore, we computed stiffness maps based on our approach for manipulator configurations when one chamber pair and two chamber pairs are pressurised separately in $x, y$ and $z$-direction. Results are shown in Fig. 6. Overall, the stiffness capability decreases along the manipulator. In the $x$-axis, the stiffness decreases $6 \sim 8$ times from the middle to the tip with maximum stiffness values of $1.17 \frac{\mathrm{N}}{\mathrm{cm}}$. When two chamber pairs are pressurised, maximum stiffness values reach $1.45 \frac{\mathrm{N}}{\mathrm{cm}}$. In the $y$-axis, maximum stiffness values only reach $0.7 \frac{\mathrm{N}}{\mathrm{cm}}$. Stiffness decreases with the increase of pressure in $y$-axis but increases in $x$-axis at a certain length of manipulator. In $z$-direction, stiffness at certain length is also increasing with higher pressures. However, stiffness variation is much higher as it ranges between $2 \sim 10$ times.

\section{CONCLUSiOnS}

We proposed a screw theory-based stiffness analysis. First, we derived the forward kinematics based on a parameterbased PCC model. It is worth noting, our stiffness analysis can be conducted based on any free-space forward kinematic model. Then our stiffness analysis and mapping methodology was conducted. Initial results of our approach demonstrate the feasibility comparing computational and experimental data. Based on our approach, information on configurations can be returned to optimise task-specific stiffness.

In future work, we will extensively collect and analyse position and stiffness data from our manipulator and explore how to scale up and apply our model to a series of soft robotic manipulators. 


\section{REFERENCES}

[1] C. Laschi, B. Mazzolai, and M. Cianchetti, "Soft robotics: Technologies and systems pushing the boundaries of robot abilities," Science Robotics, vol. 1, no. 1, p. eaah3690, 2016.

[2] A. Ataka, P. Qi, A. Shiva, A. Shafti, H. Wurdemann, H. Liu, and K. Althoefer, "Real-time pose estimation and obstacle avoidance for multi-segment continuum manipulator in dynamic environments," in IEEE/RSJ International Conference on Intelligent Robots and Systems, 2016, pp. 2827-2832.

[3] A. Arezzo, Y. Mintz, M. Allaix, S. Arolfo, M. Bonino, G. Gerboni, M. Brancadoro, M. Cianchetti, A. Menciassi, H. Wurdemann, Y. Noh, K. Althoefer, J. Fras, J. Glowka, Z. Nawrat, G. Cassidy, R. Walker, and M. Morino, "Total mesorectal excision using a soft and flexible robotic arm: a feasibility study in cadaver models," Surgical Endoscopy, vol. 31, no. 1, pp. 264-273, 2017.

[4] A. Firouzeh, M. Salerno, and J. Paik, "Stiffness control with shape memory polymer in underactuated robotic origamis," IEEE Transactions on Robotics, vol. 33, no. 4, pp. 765-777, 2017.

[5] A. Stilli, H. A. Wurdemann, and K. Althoefer, "Shrinkable, stiffnesscontrollable soft manipulator based on a bio-inspired antagonistic actuation principle," in IEEE/RSJ International Conference on Intelligent Robots and Systems, 2014, pp. 2476-2481.

[6] H. X. Trinh, V. A. Ho, and K. Shibuya, "Theoretical foundation for design of friction-tunable soft finger with wrinkle's morphology," IEEE Robotics and Automation Letters, vol. 4, no. 4, pp. 4027-4034, 2019.

[7] C. Lee, M. Kim, Y. J. Kim, N. Hong, S. Ryu, H. J. Kim, and S. Kim, "Soft robot review," International Journal of Control, Automation and Systems, vol. 15, no. 1, pp. 3-15, 2017.

[8] A. Stilli, H. A. Wurdemann, and K. Althoefer, "A novel concept for safe, stiffness-controllable robot links," Soft Robotics, vol. 4, no. 1, pp. 16-22, 2017.

[9] A. Stilli, L. Grattarola, H. Feldmann, H. A. Wurdemann, and K. Althoefer, "Variable stiffness link (VSL): Toward inherently safe robotic manipulators," in IEEE International Conference on Robotics and Automation, 2017, pp. 4971-4976.

[10] M. Cianchetti, T. Ranzani, G. Gerboni, T. Nanayakkara, K. Althoefer, P. Dasgupta, and A. Menciassi, "Soft robotics technologies to address shortcomings in today's minimally invasive surgery: the STIFF-FLOP approach," Soft Robotics, vol. 1, no. 2, pp. 122-131, 2014.

[11] H. Abidi, G. Gerboni, M. Brancadoro, J. Fras, A. Diodato, M. Cianchetti, H. Wurdemann, K. Althoefer, and A. Menciassi, "Highly dexterous 2-module soft robot for intra-organ navigation in minimally invasive surgery," The International Journal of Medical Robotics and Computer Assisted Surgery, vol. 14, no. 1, p. e1875, 2018.

[12] J. Fraś, J. Czarnowski, M. Maciaś, J. Główka, M. Cianchetti, and A. Menciassi, "New STIFF-FLOP module construction idea for improved actuation and sensing," in IEEE International Conference on Robotics and Automation, 2015, pp. 2901-2906.

[13] A. Palombi, G. M. Bosi, S. Di Giuseppe, E. De Momi, S. HomerVanniasinkam, G. Burriesci, and H. A. Wurdemann, "Sizing the aortic annulus with a robotised, commercially available soft balloon catheter: in vitro study on idealised phantoms," in International Conference on Robotics and Automation, 2019, pp. 6230-6236.

[14] A. Stilli, A. Cremoni, M. Bianchi, A. Ridolfi, F. Gerii, F. Vannetti, H. A. Wurdemann, B. Allotta, and K. Althoefer, "Airexglove - a novel pneumatic exoskeleton glove for adaptive hand rehabilitation in post-stroke patients," in IEEE International Conference on Soft Robotics, 2018, pp. 579-584.

[15] T. G. Thuruthel, M. Manti, E. Falotico, M. Cianchetti, and C. Laschi, "Induced vibrations of soft robotic manipulators for controller design and stiffness estimation," in IEEE International Conference on Biomedical Robotics and Biomechatronics, 2018, pp. 550-555.

[16] S. M. H. Sadati, A. Shiva, N. Herzig, C. D. Rucker, H. Hauser, I. D. Walker, C. Bergeles, K. Althoefer, and T. Nanayakkara, "Stiffness imaging with a continuum appendage: Real-time shape and tip force estimation from base load readings," IEEE Robotics and Automation Letters, vol. 5, no. 2, pp. 2824-2831, 2020.

[17] J. Fras, Y. Noh, H. Wurdemann, and K. Althoefer, "Soft fluidic rotary actuator with improved actuation properties," in IEEE/RSJ International Conference on Intelligent Robots and Systems, 2017, pp. 5610-5615.
[18] A. Jiang, E. Secco, H. Wurdemann, T. Nanayakkara, P. Dasgupta, and K. Athoefer, "Stiffness-controllable octopus-like robot arm for minimally invasive surgery," in Workshop on New Technologies for Computer/Robot Assisted Surgery, 2013.

[19] J. Peters, E. Nolan, M. Wiese, M. Miodownik, S. Spurgeon, A. Arezzo, A. Raatz, and H. A. Wurdemann, "Actuation and stiffening in fluiddriven soft robots using low-melting-point material," in IEEE/RSJ International Conference on Intelligent Robots and Systems, 2019, pp. 4692-4698.

[20] H. Wurdemann, A. Stilli, and K. Althoefer, "An antagonistic actuation technique for simultaneous stiffness and position control," in Intelligent Robotics and Applications, 2015, pp. 164-174.

[21] A. Shiva, A. Stilli, Y. Noh, A. Faragasso, I. De Falco, G. Gerboni, M. Cianchetti, A. Menciassi, K. Althoefer, and H. A. Wurdemann, "Tendon-based stiffening for a pneumatically actuated soft manipulator,' IEEE Robotics and Automation Letters, vol. 1, no. 2, pp. 632-637, 2016.

[22] M. Manti, V. Cacucciolo, and M. Cianchetti, "Stiffening in soft robotics: A review of the state of the art," IEEE Robotics \& Automation Magazine, vol. 23, no. 3, pp. 93-106, 2016.

[23] A. Shiva, S. H. Sadati, Y. Noh, J. Fraś, A. Ataka, H. Würdemann, H. Hauser, I. D. Walker, T. Nanayakkara, and K. Althoefer, "Elasticity versus hyperelasticity considerations in quasistatic modeling of a soft finger-like robotic appendage for real-time position and force estimation," Soft Robotics, vol. 6, no. 2, pp. 228-249, 2019.

[24] F. Renda, F. Boyer, J. Dias, and L. Seneviratne, "Discrete cosserat approach for multisection soft manipulator dynamics," IEEE Transactions on Robotics, vol. 34, no. 6, pp. 1518-1533, 2018.

[25] F. Renda, M. Cianchetti, H. Abidi, J. Dias, and L. Seneviratne, "Screw-based modeling of soft manipulators with tendon and fluidic actuation,” Journal of Mechanisms and Robotics, vol. 9, no. 4, 2017.

[26] K. Oliver-Butler, J. Till, and C. Rucker, "Continuum robot stiffness under external loads and prescribed tendon displacements," IEEE Transactions on Robotics, vol. 35, no. 2, pp. 403-419, 2019.

[27] L. Lindenroth, J. Back, A. Schoisengeier, Y. Noh, H. Würdemann, K. Althoefer, and H. Liu, "Stiffness-based modelling of a hydraulically-actuated soft robotics manipulator," in IEEE/RSJ International Conference on Intelligent Robots and Systems, 2016, pp. 2458-2463.

[28] C. Qiu, P. Qi, H. Liu, K. Althoefer, and J. S. Dai, "Six dimensional compliance analysis of ortho-planar springs for a continuum manipulator," in International Design Engineering Technical Conferences and Computers and Information in Engineering Conference, vol. 46360, 2014, p. V05AT08A036.

[29] P. Qi, C. Qiu, H. Liu, J. S. Dai, L. D. Seneviratne, and K. Althoefer, “A novel continuum manipulator design using serially connected doublelayer planar springs," IEEE/ASME Transactions on Mechatronics, vol. 21, no. 3, pp. 1281-1292, 2015.

[30] R. M. Murray, A mathematical introduction to robotic manipulation. CRC press, 2017.

[31] R. J. Webster III and B. A. Jones, "Design and kinematic modeling of constant curvature continuum robots: A review," The International Journal of Robotics Research, vol. 29, no. 13, pp. 1661-1683, 2010.

[32] J. Selig and X. Ding, "A screw theory of static beams," in IEEE/RSJ International Conference on Intelligent Robots and Systems, 2001, pp. 312-317.

[33] C. Qiu and J. S. Dai, Analysis and synthesis of compliant parallel mechanisms-Screw Theory approach. Springer, 2020. 